\title{
O Organizar de Práticas Cooperativas no Contexto de um Ambiente de Estágio em Desenvolvimento de Software
}

\author{
Ariádna Miranda ${ }^{1}$, Allysson Allex Araújo ${ }^{1}$, Emanuel Coutinho ${ }^{1}$ e Jerffeson Souza ${ }^{2}$ \\ ${ }^{1}$ Universidade Federal do Ceará (UFC) \\ Grupo de Estudos em Sistemas de Informação e Inovação Digital (GESID) \\ ${ }^{2}$ Universidade Estadual do Ceará (UECE) \\ Programa de Pós-graduação em Ciência da Computação
}

\begin{abstract}
Soft skills are not trivial to be taught, although they are preponderant to be improved during the academic experience of future software engineers. Understanding how such skills are perceived and practiced during the supervised internship poses to be relevant, given the opportunity for students to enhance their skills. Grounded on the Practice Theory, we investigate how the organization of soft skills occurs as a bundle of cooperative practices in the context of the Federal University of Ceará (Campus de Crateús) internship environment. We highlight as contributions $i$ ) the proposition of a conceptual model for investigating cooperative practices; ii) the analytical contrast between doings and sayings and iii) the empirical reflection for students on the role of soft skills.
\end{abstract}

Resumo. As soft skills não são triviais de serem ensinadas, embora sejam preponderantes de serem aprimoradas durante a formação acadêmica de futuros engenheiros de software. Compreender como tais habilidades são percebidas e exercidas durante o estágio supervisionado demonstra-se salutar, tendo em vista a oportunidade de lapidação formativa dos discentes. Sob a lente da Teoria das Práticas, esta pesquisa investiga como ocorre o organizar de soft skills enquanto feixe de práticas cooperativas no ambiente de estágio da Universidade Federal do Ceará (Campus de Crateús). Dentre as contribuições, destaca-se i) a proposição de um modelo conceitual para investigação das práticas cooperativas; ii) contraste analítico entre feitos e ditos e iii) viabilização de reflexão empırica aos alunos sobre o papel das soft skills.

\section{Introdução}

As soft skills refletem competências usadas pelos indivíduos ao interagir com outros indivíduos e, consequentemente, podem ser desenvolvidas e aprimoradas através da experiência [Hubbard 2012]. Todavia, as soft skills não são habilidades triviais de serem ensinadas, embora sejam preponderantes de serem praticadas e aprimoradas durante a formação acadêmica [Martins 2017]. Nesse contexto, percebe-se a cooperação durante o processo de desenvolvimento de maneira tão importante quanto as questões técnicas [de Souza et al. 2009], haja vista que software são criados por e para pessoas, e entender os aspectos cooperativos e humanos demonstra-se crucial para compreender como aprimorar o uso de ferramentas e métodos [Prikladnicki et al. 2013].

Através de uma lente socio-construtivista, a Teoria das Práticas busca compreender como as organizações acontecem além da lógica funcional [Ipiranga and Lopes 2016]. 
Em suma, tal teoria propõe que indivíduos influenciam e são influenciados em uma complexa teia de relações mútuas [Latour 2005]. Essa rede social, denominada tecido social, é constituída pelos indivíduos e pelas múltiplas formas de laços sociais que os interligam [Elias 1994]. Logo, compreende-se as organizações não mais feitas de forma rígida e formal, mas sim como uma entidade que está em constante transformação através das práticas, ou seja, um conjunto incorporado de "feitos" (doings) e "ditos" (sayings) [Schatzki and Schatzki 1996]. Todavia, apesar das práticas terem sido previamente investigadas sob a ótica da Engenharia de Software [Giuffrida and Dittrich 2014, Päivärinta and Smolander 2015], identifica-se, em especial, uma lacuna concernente ao estudo das práticas de cooperação sob a ótica das soft skills num ambiente de estágio em desenvolvimento de software. Em particular, justifica-se o contexto de estágio como pertinente tendo em vista sua responsabilidade enquanto mecanismo para lapidação formativa e a demanda do mercado em relação ao aprimoramento de soft skills [Ahmed et al. 2015].

Alinhado a motivação delineada previamente, esta pesquisa assume um caminho metodológico exploratório para compreender, através de um estudo de caso, as práticas de cooperação exercidas por alunos no contexto do ambiente de estágio da Universidade Federal do Ceará (Campus de Crateús). Norteia-se o escopo analítico através de um enfoque qualitativo com base na seguinte questão de pesquisa: como ocorre o organizar de soft skills enquanto feixe de práticas cooperativas no contexto de um ambiente de estágio em desenvolvimento de software? Dentre as contribuições, destaca-se i) a proposição de um modelo conceitual para investigação das práticas cooperativas em ambiente de estágio; ii) contraste entre feitos e ditos quanto à epistemologia das práticas de cooperação realizadas pelos discentes e iii) viabilização de reflexão empırica aos alunos sobre soft skills.

\section{Práticas em Engenharia de Software}

O movimento epistemológico Practice Turn tem salientado a importância sobre o estudo das práticas nas organizações, inclusive na tecnologia [Cetina et al. 2005]. Como intrínseca às atividades humanas, compreende-se a prática (praktik) como um "tipo de comportamento rotineiro que consiste em vários elementos, interconectados entre si: formas de atividades corporais, formas de atividades mentais, 'coisas' e seu uso, um conhecimento de fundo na forma de compreensão, know-how, estados de emoção e motivação conhecimento" [Reckwitz 2002]. Assim, as práticas são um conjunto organizado e amplo de articulações de ações intercaladas compostas por um conjunto de "feitos" e "ditos" [Schatzki and Schatzki 1996, Schatzki 2005]. Tais feitos e ditos são incorporados como o conjunto de ações consideradas básicas feitas com o corpo, não sendo apenas padrões de comportamento, mas formas de como fazer algo [Ipiranga and Lopes 2016].

Segundo Schatzki (2005), as ações das pessoas são interligadas não de uma maneira desordenada, mas na forma de uma estrutura de governança composta por três elementos fundamentais. Primeiramente, os entendimentos referem-se à habilidade de saber fazer algo, ou seja, como algo pode ser feito e como identificar e instigar as ações nos outros. Por sua vez, as regras referem-se às formulações explícitas, instruções, preceitos que direcionam e instruem o que deve ser feito ou dito. As regras fazem surgir, impedir ou provocar novas ações ou ainda controlam atividades existentes. Finalmente, as estruturas teleoafetivas são um conjunto hierarquicamente organizado e normativo de fins (objetivos e interesses) para alcançar meios, como projetos, ações e emoções considerados válidos na prática. O termo normativo não se refere somente a determinar que uma 
ação seja certa ou errada, mas indicar que algumas delas podem ser aceitáveis.

De acordo com Dittrich et al. (2002), as práticas de software ocorrem durante o desenvolvimento ou uso do software, inclusive através da adaptação da tecnologia e a forma como as pessoas se relacionam. O desenvolvimento de software requer várias práticas, incluindo análise, design, implementação e gerenciamento de qualidade [Päivärinta and Smolander 2015]. Dittrich (2016) salienta a relevância em ter uma base teórica para entender o desenvolvimento de software como prática e para se compreender como os métodos e ferramentas são apropriados. Portanto, afirmar que a Engenharia de Software é uma prática é ressaltar que ela possui atividades coordenadas e conectadas através de estruturas teleoafetivas compartilhadas, pois a intenção é projetar um software; entendimentos compartilhados que consistem em desenvolver software tanto no geral ou como um domínio específico, ou como parte de uma organização; e por último, regras através de manuais, ou ferramentas/métodos aprendidos em capacitações [Dittrich 2016].

\section{Procedimentos Metodológicos}

O caminho metodológico trilhado, de natureza exploratória, enquadrou-se num escopo de análise qualitativa devido a necessidade de compreender elementos subjetivos adjacentes ao fenômeno. Assim, investigou-se em profundidade um estudo de caso único e instrumental buscando resultados além do caso examinado em prol de uma compreensão teórica ampla [Yin 2015]. Fundamentado em [Stake 2005], este trabalho não objetiva alcançar validade externa, mas plausibilidade na construção de uma generalização analítica. O estudo de caso explorado envolveu o ambiente de estágio da Universidade Federal do Ceará (Campus de Crateús), o qual é composto pelo Núcleo de Prática em Desenvolvimento de Sistemas (NPDS) e o Ecossistema Parque Tecnológico de Crateús (SParC). O NPDS objetiva prover aos estudantes um ambiente para desenvolvimento de projetos de tecnologia, contando atualmente com 5 estagiários, 3 projetos e 2 professores supervisores presenciais. Enquanto o SParC é um centro de fomento a pesquisa e empreendedorismo que dispõe atualmente de 4 estagiários, 2 projetos e 2 supervisores remotos.

O plano metodológico foi organizado em três macro-etapas. Numa primeira etapa realizou-se uma análise de dados secundários através de pesquisa bibliográfica em prol da compreensão de categorias teóricas pertinentes ao estudo. A partir dessa compreensão, elaborou-se um modelo conceitual para apoiar a investigação das práticas cooperativas no ambiente sob análise. Na segunda etapa, referente à coleta de dados primários, adotou-se duas técnicas. Inicialmente, utilizou-se a coleta de dados através de Observação Direta focando-se na perspectiva dos "feitos". Em seguida, iniciou-se as Entrevistas em Profundidade com os estagiários em busca dos "ditos". As temáticas abordadas nos roteiros de entrevistas foram estabelecidas a partir das categorias teóricas sobre soft skills e cooperação em desenvolvimento de software obtidas na primeira etapa. Finalmente, na terceira etapa, realizou-se a análise qualitativa das informações coletadas. Nessa etapa, optou-se pela técnica da Análise Temática de Conteúdo [Bardin 1979], cujo objetivo consiste em destacar os itens de significação a partir da descrição do corpus obtido. Com isso, possibilita-se contrastar os ditos e feitos ao reinterpretar mensagens e significados.

Em relação ao protocolo na coleta de dados, destaca-se que a Observação Direta ocorreu no ambiente de estágio dos alunos visando compreender as interações relacionadas às categorias teóricas sobre soft skills e cooperação identificadas na Etapa 1. A 
permanência no campo ocorreu nos dias úteis entre 30 de setembro de 2019 e 30 de outubro de 2019, durando em média 4 horas por dia (no período da manhã). As observações foram registradas em diário de campo durante duas sprints, a qual cada uma correspondeu a 15 dias. Ao final de cada sprint, é verificado pelo professor supervisor se as tarefas estão sendo realizadas e se estão de acordo com requisitos especificados. As Entrevistas em Profundidade foram feitas individualmente e presencialmente com cada um dos dez estagiários, os quais apresentam idade entre 19 e 23 anos, sendo todos estudantes do curso de Ciência da Computação e alocados em projetos de tecnologia. Cinco deles estão no estágio entre 6 e 12 meses, e os outros cinco estão entre 3 a 4 meses. A Tabela 1 apresenta uma síntese caracterizando os participantes cujos nomes foram omitidos para preservar a confidencialidade. O questionário foi dividido em 5 seções onde buscou-se caracterizar os participantes, bem como nortear a perguntas à luz do modelo conceitual previamente estabelecido. Detalhes sobre as entrevistas encontram-se disponíveis na página de suporte ${ }^{1}$.

\begin{tabular}{l|l|l|l|l|l} 
Entrevistado & Idade & Tempo de estágio & Projeto & Ambiente de estágio & Supervisores \\
\hline E1 & 21 anos & 10 meses & A & SParC & S3 \\
\hline E2 & 20 anos & 6 meses & E & NPDS & S1 e S2 \\
\hline E3 & 23 anos & 3 meses & E & NPDS & S1 e S2 \\
\hline E4 & 20 anos & 10 meses & D & NPDS & S1 e S2 \\
\hline E5 & 23 anos & 12 meses & B & SParC & S4 \\
\hline E6 & 19 anos & 3 meses & D & NPDS & S1 e S2 \\
\hline E7 & 20 anos & 3 meses & D & NPDS & S1 e S2 \\
\hline E8 & 22 anos & 5 meses & B & SParC & S4 \\
\hline E9 & 21 anos & 10 meses & C & NPDS & S1 e S2 \\
\hline E10 & 21 anos & 11 meses & A & SParC & S3
\end{tabular}

Tabela 1. Dados dos participantes.

Em relação a análise dos dados, verificou-se que as gravações dos áudios obtidos nas entrevistas e as anotações das observações refletem uma variedade rica de discussões. Com base em [Braun and Clarke 2006] e [Bardin 1979], dois autores buscaram a familiarização com os dados através da transcrição das entrevistas e a leitura e releitura das anotações feitas durante as observações. Em seguida, objetivou-se a geração sistemática de códigos, identificando indutivamente os núcleos de sentido (palavras, expressões, frases) associados às unidades de contexto (discursos) que emergiram dos temas investigados. Posteriormente, realizou-se uma revisão geral do material, para, finalmente, conduzir a análise através dos significados inferenciais e interpretativos obtidos.

\section{Modelo Conceitual}

O desenvolvimento de software é, em sua essência, cooperativo, justamente por ser um trabalho realizado de forma colaborativa pelas pessoas [Saeki 1995]. A falta de cooperação pode apresentar diversos problemas como, por exemplo, menor produtividade [Tessem and Iden 2008]. Dentre as soft skills mais requisitadas no mercado de software, pode-se destacar trabalho em equipe, liderança e comunicação [Ahmed et al. 2015].

Moe et al. (2009) definem trabalho em equipe como um grupo de pessoas com habilidades complementares em busca de um objetivo comum. A cooperação, coordenação e abordagens coletivas para o trabalho são características desejáveis para o trabalho em equipe [Janz 1999], em especial no contexto de desenvolvimento de software. A liderança, por sua vez, é outro fator crucial para o sucesso de projetos de software [Faraj and Sambamurthy 2006]. Assim, torna-se essencial estabelecer habilidades

\footnotetext{
${ }^{1}$ http://gesid.github.io/papers/soft-skills-estagio
} 
de liderança em equipes multidisciplinares que demandam habilidades de coordenação [Futrell et al. 2001]. De acordo com Botelho e Krom (2010), existe uma distinção entre líder e estilo de liderança. Líder é a pessoa a qual foi atribuída a responsabilidade de dirigir, e estilo de liderança é a maneira pelo qual o líder influencia pessoas, que pode ser autocrático (toma decisões sem consultar o grupo), democrático (compartilha do time na decisão) e liberal (só intervém quando solicitado). Ademais, sabe-se que a falta de comunicação afeta a equipe como um todo, pois dificulta o compartilhamento de informações e experiências. Logo, a qualidade da comunicação dentro de uma equipe se define em frequência (regularidade e tempo) e formalização (documentado ou espontânea) da troca de informações [Pinto and Pinto 1990].

Abstraindo os elementos teóricos previamente delineados, propõe-se um modelo conceitual (Figura 1) o qual fundamenta-se na concepção de que as soft skills (liderança, comunicação e trabalho em equipe) se relacionam como feixes de práticas cooperativas. Essas habilidades manifestam-se através de práticas caracterizadas por Entendimentos, Regras e Estruturas Teleoafetivas visando potencializar a produtividade do time. Estes conjuntos abertos de ditos e feitos são organizados pelas práticas relacionadas aos Entendimentos sobre essas ações, por meio das Regras que são repassadas aos praticantes, e pelas Estruturas Teleoafetivas que se constituem como uma matriz de elementos diversos (formas de fazer, objetivos, emoções, etc) [Schatzki 2005]. Esses fazeres e dizeres, que pertencem a uma determinada prática, se expressam como componentes da organização da prática, implicando, assim, nesta delimitação de fronteiras sob uma sobreposição espacial e temporal [Ipiranga and Lopes 2016]. Finalmente, define-se um mecanismo de Feedback de Aprendizado a partir da relação entre as práticas e produtividade, fomentando, assim, uma cultura cíclica de melhoria contínua das práticas na organização.

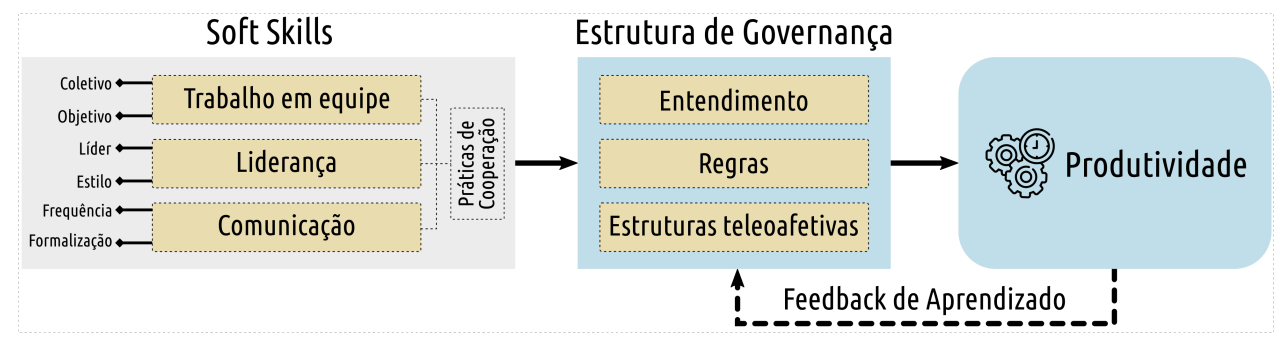

Figura 1. Proposta de Modelo Conceitual.

\section{Análises e Resultados}

Analisa-se a seguir a percepção dos alunos sobre soft skills e, posteriormente, o contraste entre feitos e ditos quanto às práticas relacionadas aos construtos liderança, comunicação e trabalho em equipe a partir dos Entendimentos, Regras e Estruturas Teleoafetivas.

\subsection{Percepção dos estagiários sobre soft skills}

Indagou-se aos participantes sobre as dificuldades que eles enfrentaram no estágio. Oito discentes destacaram a adaptação com a nova rotina, pois tinham que estar na Universidade mais cedo. Também salientaram o desafio quanto ao aprendizado das tecnologias adotadas. Tais questões demandavam um sentimento de compromisso e adaptação. Sobre o papel das soft skills no desenvolvimento de software, observou-se que seis entrevistados (E1, E3, E4, E7, E8 e E9) declararam não saber formalmente do que se tratavam as 
soft skills, refletindo, assim, pouco contato com o desenvolvimento de habilidades interpessoais instrucionalmente. Outros alunos demonstraram reconhecimento sobre o tema. E6, por exemplo, destacou: "É algo totalmente necessário e que muitas vezes as pessoas não dão tanta importância, porém não adianta o programador dominar as tecnologias e não saber trabalhar em equipe". Outra manifestação emblemática foi proferida por E2: "Não é tão necessária, pois a maioria dos trabalhos hoje em dia é remoto, aí não precisa tanto dessas soft skills". Apesar de indicar que supostamente reconhece soft skills, tal fala sugere incompreensão prática. Adicionalmente, questionou-se, numa escala de 0 a 5, sobre quão preparados os alunos julgavam estar quanto ao desenvolvimento de soft skills. Sete estudantes consideram como "médio" (3), um como "adequado" (4) e dois como "muito adequado" (5). Em relação ao preparo, E3 afirmou: "Eu me preparei antes mesmo de conhecer o ambiente de desenvolvimento de software. Eu já trabalhava muito a comunicação entre pessoas, tanto que já fiz vários cursos de comunicação interpessoal".

\subsection{Liderança}

As interpretações dos Entendimentos e Regras advém do que os estagiários definem como liderança e quais práticas eles julgam que um bom líder deve exercer. Em termos gerais, constatou-se que os entrevistados convergiram para a concepção de líder como um componente fundamental da equipe, que tem capacidade de guiá-la, estipulando tarefas para a melhor evolução do projeto. E3 salienta o líder como "Componente da equipe capaz de guiar o time para melhor direcionamento no desenvolvimento do projeto". E8 pondera a necessidade de muitas vezes, mesmo não sendo o supervisor, um dos estudantes assumir essa posição tomando decisões fundamentais. Os discentes mencionaram com frequência a ideia de que liderança "não é apenas mandar". Para eles, a liderança não deve ser autocrática, mas sim democrática. Interessante destacar o caso do Projeto $\mathrm{B}$ o qual é supervisionado remotamente pelo supervisor S4. E5, alocado ao referido projeto, relatou a necessidade no compartilhamento da liderança. Segundo ele, “(...) em alguns momentos é necessário ter uma liderança informal (...) que muitas vezes eu assumo aqui, mas eu não acho que eu tenha total capacidade. Em outros momentos são meus colegas".

Quanto a liderança observada, ou seja, em relação aos "feitos", identificou-se as lideranças formais exercidas pelos professores. Notou-se que S1 executa práticas de liderança democrática, enquanto S2 enquadra-se como liberal. S1 demonstrava constantemente interesse nas tarefas feitas pelos estagiários, revisando-as diariamente com os discentes em suas respectivas mesas. O supervisor S2 apenas intervinha quando solicitado, apresentando, assim, menos frequência de atendimento. Por sua vez, os professores S3 e S4 atuam de forma remota, mas, através da observação da execução dos projetos, identifica-se ambos como democráticos.

Quando questionados sobre as práticas que um bom líder deve exercer, surgiram respostas relacionadas ao ato de inspirar a equipe, zelar pelo respeito e aprimorar a comunicação. Quanto ao respeito, E2 enfatizou: "tem líder que está na vaga de líder, mas não respeita os outros". Três alunos (E1, E3 e E7) reforçaram que um líder deve estar interessado na evolução do projeto, acompanhando se alguém está com dificuldade. Ao serem indagados sobre como se enxergam exercendo as práticas de um bom líder, oito deles (exceção de E5 e E10), ou seja, a grande maioria, não se considera com perfil de líder e não se sente seguro para exercer atividades de liderança. Ao serem questionados se percebiam algum colega com esse perfil, cinco deles citaram alguém, sendo E10 
o mais lembrado. E4 salienta a importância quanto ao comportamento de E10 em tirar dúvidas dos demais: "O E10 ajudava todo mundo, tirava dúvidas". Constata-se que a liderança entre os colegas está associada a cooperação quando há dificuldades, principalmente técnicas. Percebeu-se que, ao sentirem dificuldades em dada tarefa, os alunos frequentemente recorriam a algum membro do projeto ou, inclusive, de outro.

No que se refere às Estruturas Teleoafetivas, as principais falas envolveram os impactos positivos da liderança sobre a produtividade. De acordo com E2, E4 e E5, há um esforço maior no cumprimento das tarefas quando há um líder presente. E5 salienta que consegue-se "Extrair da gente aquilo que a gente precisa fazer"e discorre que muitas vezes ocorre acomodação por parte de alguns alunos na realização das tarefas e um bom líder incentiva a buscar o conhecimento. Observou-se também uma excessiva dependência dos alunos quanto a figura do supervisor do projeto, que, por muitas vezes, acabava atrasando a evolução do projeto em decorrência das demandas que o professor lidava. Em síntese, na visão dos estagiários, um bom líder deve exercer práticas de motivação e incentivo de modo a possibilitar que os projetos sejam mais produtivos.

\subsection{Comunicação}

Quanto aos Entendimentos, percebeu-se "passar e entender a mensagem, de forma simples e direta" como síntese de comunicação adequada. E5 destacou como uma qualidade "Saber criar um diálogo, saber passar sua ideia", enquanto E8 relatou seu entendimento da seguinte forma: "Quando eu consigo passar o que quero passar e quando eu consigo entender o que alguém está tentando dizer". Quando questionados sobre as dificuldades em relação à comunicação, oito estagiários (exceção dos alunos E2 e E5) convergiram para a concepção de não terem enfrentado muita dificuldade, pois maioria se conhece previamente e apresenta um bom relacionamento fora do estágio. E5, porém, relatou uma dificuldade quanto ao seu colega, o qual alegou nunca ter conversado previamente, tampouco serem amigos fora do estágio. Segundo ele, isso atrapalhou o andamento do projeto, pois, inclusive, chegaram a não entregar tarefas pela ausência de uma comunicação. Quando contestados sobre o que fazem quando enfrentam dificuldades, os discentes relataram que, além de pesquisar na internet, procuram se comunicar com algum colega que esteja próximo, seja do seu ou de outro projeto. Todos os estagiários alegaram que a comunicação no ambiente poderia melhorar em relação aos supervisores, pois, entre os alunos estava satisfatória. Os discentes identificaram que S1 é mais comunicativo do que S2. De acordo com os alunos, o S1 demonstra-se mais comunicativo, enquanto sentem falta de uma participação maior de $\mathrm{S} 2$. Os estagiários que trabalham sob supervisão remota, ou seja, com S3 e S4, também alegam dificuldades quanto a demora de retorno.

À luz das Regras, identificou-se que, para a comunicação ocorrer, é importante que ela seja feita de forma clara. Quando questionados sobre adoção de meios para comunicação formal, relatou-se o uso de documentações e realização de reuniões. Foi mencionado e observado o uso de meios digitais como WhatsApp, Telegram, Gitlab e e-mail (mais utilizado para envio de relatórios). O uso do Gitlab demonstrou-se frequentemente útil em termos de comunicação para os times, pois, através dessa ferramenta, os alunos sabiam informações sobre a sprint. Através do WhatsApp, por sua vez, havia uma comunicação mais dinâmica, onde os supervisores repassavam informes e oportunidades de emprego. Observou-se, entretanto, que a comunicação ocorre, em grande medida, de modo informal, haja vista que a maioria do discentes demonstram-se próximos fora do 
ambiente de estágio. Tal informalidade favorece interações amistosas e o fortalecimento das relações sociais. Entretanto, percebeu-se um acanhamento de contato dos alunos em relação aos supervisores, especialmente quando não estavam presentes fisicamente.

Em termos de Estruturas Teleoafetivas, constatou-se uma percepção geral dos alunos sobre o impacto positivo que uma boa comunicação exerce sobre a produtividade. De acordo com E2, quanto mais se comunica com o líder para tirar dúvidas em relação à atividade que se pretende fazer, mais ficará claro e não haverá perda de tempo com relação a entrega de tarefas, aumentando, assim, a produtividade. Isso reflete uma preocupação em evitar retrabalho. Três estagiários (E1, E4 e E5) mencionaram a comunicação como um ponto positivo nesse sentido. Verificou-se que a presença frequente do S1, discutindo os projetos com cada equipe, favorecia bastante em mitigar o retrabalho.

\subsection{Trabalho em Equipe}

Visando interpretar os Entendimentos, questionou-se aos alunos como eles compreendem o trabalho em equipe. E6, E9 e E10 refletiram sobre conscientizar que lidam com pessoas com características diferentes e que demanda-se adaptação. Outra prática mencionada é a divisão de tarefas, pois agiliza o que tem que ser feito. Para os discentes, faz-se necessário que todos estejam alinhados ao projeto, ou seja, que saibam o que está sendo feito e qual o objetivo. Ao serem indagados sobre o que entendem por cooperação, as definições estavam ligadas ao trabalho em equipe. E3 concebeu: "Junção de um trabalho em equipe com a dedicação de manter esse trabalho". E4 reforçou o sentimento de união para resolução de uma tarefa: "É uma coisa ligada totalmente ao trabalho em equipe (...), seria trabalhar junto para resolver determinada tarefa". E6 salientou que é "Estar alinhado com o grupo (...), entender que o grupo tem um propósito". Ao serem provocados sobre o papel deles em relação à cooperação, 8 estagiários assumiram sempre cooperar e ajudar os colegas com dificuldades. Apenas dois colegas (E4 e E2) mencionaram que não se consideram muito cooperativos, pela insegurança em ajudar os colegas. Tal insegurança é manifestada por E4: "Poderia ser maior (a cooperação), alguns problemas eu sei resolver mas pela insegurança que eu tenho, prefiro dizer que eu não sei".

Ao observar o cotidiano, percebeu-se, em especial, que os membros do Projeto A demonstravam alto engajamento. Tal particularidade advém dos estagiários apresentarem uma relação amigável, facilitando a interação. Nenhum dos entrevistados relatou sentir dificuldades quanto ao exercício do trabalho em equipe atualmente. Dois estagiários ponderaram que, no início, sentiram dificuldades, por haver barreira de comunicação. E2 mencionou: "Atualmente não, antigamente sim. Porque meu colega era tão ruim de se comunicar quanto eu", enquanto E1 relatou ter tido um período de adaptação com S3, por mais que tivesse cursado disciplinas com o mesmo, ainda sentia-se tímido. Para facilitar o trabalho em equipe, observou-se a adoção de métodos e ferramentas como o Gitlab para alocação de tarefas, grupos de WhatsApp para comunicação, e a metodologia ágil Scrum para gerenciamento dos projetos. Quanto aos projetos coordenados remotamente, há ampla adoção do Hangouts e comunicação direta via WhatsApp.

Ao interpretar as Regras, verificou-se a adoção dos ritos de agilidade oriundos do Scrum, vide reuniões diárias e timeboxing. Todos os estagiários destacaram a boa comunicação como aspecto fundamental, inclusive via comentário em código-fonte. E5 salientou: "Vejo uma vantagem muito grande quando eu faço um código pensando em 
como meu colega de trabalho vai ver aquilo ali e ele vai entender (...) eu sei como ele trabalha". E7 e E9 mencionaram a importância em cada membro trabalhar de forma igualitária, de tal modo que não sobrecarregue ninguém. Alinhado a tal premissa, os estagiários contextualizaram a importância quanto a responsabilidade, principalmente em relação ao que foi atribuído. Uma fala que se reverberou bastante foi "Manter o respeito". Para eles, há de se manter o respeito entre os membros, incluindo os líderes formais.

Quanto às Estruturas Teleoafetivas, investigou-se os impactos positivos relacionados à produtividade decorrente do trabalho em equipe. Segundo E3, "Quando há um trabalho em equipe, a produtividade é bastante efetiva". E10 complementa ao destacar que "Na produtividade, o trabalho em equipe é essencial". Para eles, se não houver trabalho em equipe, as tarefas levam mais tempo para serem concluídas e, consequentemente, a produtividade diminui. E8 discerne que "Toda a equipe se complementa", demonstrando, assim, reconhecimento sobre a multidisciplinaridade do time. De forma geral, observou-se ampla adoção de práticas cooperativas entre os discentes e, inclusive, para com o supervisores, ao unirem esforços para resolução dos desafios que surgiam.

\section{Considerações Finais}

Usufruindo da epistemologia das práticas sob um enfoque qualitativo, este estudo apresentou o organizar de soft skills enquanto feixe de práticas cooperativas no contexto de um ambiente de estágio em desenvolvimento de software da Universidade Federal do Ceará (Campus de Crateús). Percebeu-se três principais elementos como Feedbacks de Aprendizado: 1) os alunos não percebem a si próprios como líderes, demonstrando, assim, necessidade de fortalecimento no exercício de tais práticas de forma compartilhada; 2) quanto a comunicação, destaca-se a oportunidade de potencializar a troca de conhecimento entre os membros, bem como mitigar desafios de introspecção através de dinâmicas específicas; 3) sobre trabalho em equipe, há de se refletir acerca de estratégias que incentivem a proatividade e a conscientização sobre soft skills no cotidiano. Em relação às limitações, pode-se considerar o período de observações reduzido, porém a triangulação de dados fortalece a validade dos resultados. Apesar da quantidade reduzida de alunos, destaca-se a profundidade analítica e a qualificação do contexto sob investigação. Como trabalhos futuros, pretende-se investigar outros locais e organizar um guia de boas práticas sobre soft skills em ambientes de estágio em desenvolvimento de software.

\section{Referências}

Ahmed, F., Capretz, L. F., Bouktif, S., and Campbell, P. (2015). Soft skills and software development: A reflection from the software industry. arXiv preprint arXiv:1507.06873.

Bardin, L. (1979). Análise de conteúdo. Lisboa: Edições.

Botelho, J. C. and Krom, V. (2010). Os estilos de lideranças nas organizações. XIV Encontro Latino Americano de Iniciação Científica e X Encontro Latino Americano de Pós-Graduação.

Braun, V. and Clarke, V. (2006). Using thematic analysis in psychology. qualitative research in psychology. Qualitative Research in Psychology, 3(2):77-101.

Cetina, K. K., Schatzki, T. R., and Von Savigny, E. (2005). The practice turn in contemporary theory. Routledge.

de Souza, C. R., Sharp, H., Singer, J., Cheng, L.-T., and Venolia, G. (2009). Guest editors' introduction: Cooperative and human aspects of software engineering. IEEE software, 26(6):17-19. 
Dittrich, Y. (2016). What does it mean to use a method? towards a practice theory for software engineering. Information and Software Technology, 70:220-231.

Dittrich, Y., Floyd, C., and Klischewski, R. (2002). Social Thinking-Software Practice. Mit Press.

Elias, N. (1994). A Sociedade dos Indivíduos. Zahar.

Faraj, S. and Sambamurthy, V. (2006). Leadership of information systems development projects. IEEE Transactions on engineering management, 53(2):238-249.

Futrell, R. T., Shafer, L. I., and Shafer, D. F. (2001). Quality software project management. Prentice Hall.

Giuffrida, R. and Dittrich, Y. (2014). How social software supports cooperative practices in a globally distributed software project. In Workshop on Cooperative and Human Aspects of Software Engineering, pages 24-31. ACM.

Hubbard, E.-M. (2012). 9.6. 2 developing systems engineering competencies in undergraduate students for industrial placements. In INCOSE International Symposium, volume 22, pages 1379-1386. Wiley Online Library.

Ipiranga, A. S. R. and Lopes, L. L. S. (2016). A epistemologia do campo aberto e o organizar das práticas de espaço. In Anais do Congresso Brasileiro de Estudos Organizacionais.

Janz, B. D. (1999). Self-directed teams in is: correlates for improved systems development work outcomes. Information \& Management, 35(3):171-192.

Latour, B. (2005). Reassembling the social. Politica y Sociedad, 43(3):127-130.

Martins, J. C. C. (2017). Soft Skills: conheça as ferramentas para você adquirir, consolidar e compartilhar conhecimentos. Brasport.

Moe, N. B., Dingsøyr, T., and Røyrvik, E. A. (2009). Putting agile teamwork to the test-an preliminary instrument for empirically assessing and improving agile software development. In International Conference on Agile Processes and Extreme Programming in Software Engineering, pages 114-123. Springer.

Päivärinta, T. and Smolander, K. (2015). Theorizing about software development practices. Science of Computer Programming, 101:124-135.

Pinto, M. B. and Pinto, J. K. (1990). Project team communication and cross-functional cooperation in new program development. Journal of Product Innovation Management, 7(3):200-212.

Prikladnicki, R., Dittrich, Y., Sharp, H., De Souza, C., Cataldo, M., and Hoda, R. (2013). Cooperative and human aspects of software engineering. ACM SIGSOFT Software Engineering Notes, 38(5):34-37.

Reckwitz, A. (2002). Toward a theory of social practices: A development in culturalist theorizing. European journal of social theory, 5(2):243-263.

Saeki, M. (1995). Communication, collaboration and cooperation in software development-how should we support group work in software development? In Asia Pacific Software Engineering Conference, pages 12-20. IEEE.

Schatzki, T. R. (2005). Peripheral vision: The sites of organizations. Organization studies, 26(3):465-484.

Schatzki, T. R. and Schatzki, T. R. (1996). Social practices: A Wittgensteinian approach to human activity and the social. Cambridge University Press.

Stake, R. E. (2005). Qualitative case studies. Sage Publications.

Tessem, B. and Iden, J. (2008). Cooperation between developers and operations in software engineering projects. In Workshop on cooperative and human aspects of software engineering, pages 105-108. ACM.

Yin, R. K. (2015). Estudo de Caso: Planejamento e métodos. Bookman editora. 